Psychotherapeut 2021 $66: 186-194$ https://doi.org/10.1007/s00278-021-00505-6 Angenommen: 21. Januar 2021 Online publiziert: 24. März 2021 (c) Springer Medizin Verlag $\mathrm{GmbH}$, ein Teil von Springer Nature 2021, korrigierte Publikation 2021

Die momentan wie am Fließband produzierten Befunde zu psychosozialen Aspekten der durch die "coronavirus disease 2019" (COVID19) ausgelösten Pandemie bestätigen wissenschaftliche Erkenntnisse, die bereits vor dem Ausbruch prinzipiell bekannt waren. Dabei kommt Befunden der Gruppenpsychologie und -dynamik sowohl für das Verständnis von Verhaltensweisen in der Bevölkerung wie auch für die Planung von Schutzmaßnahmen der öffentlichen Gesundheit große Bedeutung zu. Dieses Wissen und die Kompetenz wurden bislang für Entscheidungsfindungen auf dem Feld der Gesundheitspolitik und der öffentlichen Gesundheit allerdings nicht genutzt.

\section{Bedeutung von COVID-19 für die öffentliche Gesundheit (Public Health)}

Im Frühjahr 2020 strahlte das ZDF eine 8-teilige fiktionale Fernsehserie aus mit dem Titel „Sløborn“: Darin geht es um ...

eine erfundene Nordsee-Insel, an deren Küste ein Segelboot mit einem toten Weltenbummlerehepaar angetrieben wird. Noch bevor die Polizei das Boot sichern kann, entdecken es Jugendliche und ent-

Bernhard Strauß - Jenny Rosendahl - Uwe Berger

Institut für Psychosoziale Medizin, Psychotherapie und Psychoonkologie, Universitätsklinikum Jena, Jena, Deutschland

\title{
Bedeutung der COVID-19- Pandemie für die öffentliche Gesundheit und gruppenpsychologische Aspekte - Teil 2 einer (vorläufigen) Übersicht
}

wenden einige Wertgegenstände. Dabei kommen Sie auch den Toten sehr nahe. Wie sich herausstellt, war das Ehepaar mit dem im Film so genannten Taubengrippevirus infiziert, das irgendwo am anderen Ende der Welt von Tauben auf den Menschen übertragen wurde. Während der Informationsfluss vom Ursprungsort in den Rest der Welt anfangs zögerlich ist, verbreitet sich das Virus auf Sløborn. Da das Taubengrippevirus tödlich ist, werden rigide Schutzmaßnahmen eingeführt, wie Pflicht zum Tragen eines Mund-NasenSchutzes, Kontaktverbote und Quarantäne. Diese Maßnahmen stoßen jedoch rasch auf den Widerstand, z. B. von feierfreudigen Jugendlichen, freiheitsliebenden Inselbewohnern und auf der Insel festgehaltenen Touristen. Zudem formiert sich eine Gruppe, die vehement nicht an das Virus glaubt. Schließlich riegelt die Bundesregierung die Insel vom Rest der Welt ab, und Schutzmaßnahmen werden von der Bundeswehr durchgesetzt. Bereits infizierte Personen werden auf Spezialstationen außerhalb der Insel geschafft, die aber keine Kliniken sind, sondern reine Verwahranstalten, in denen die Infizierten isoliert werden und sterben. Auffällig ist die Hilflosigkeit der Regierung, die von Anfang an keinen weitsichtigen Plan zum Umgang mit dem nach und nach pandemischen Virus hat, sondern lediglich auf aktuelle Herausforderungen mit mehr oder weniger untauglichen Mitteln reagiert.

Bis aufdie Tödlichkeit des Virus, die Wendung mit den Verwahranstalten und den Einsatz der Bundeswehr zur gewaltsamen Durchsetzung der Regierungsmaßnahmen ist die Serie unglaublich nah an dem, was nach Ausbruch der COVID19-Pandemie weltweit passierte. Aber das wirklich Erstaunliche ist, dass das Drehbuch (ebenso wie in dem Buch zur Psychologie der Pandemie von Taylor (2020)) bereits im Herbst 2019 fertig und die Serie bereits komplett abgedreht war, bevor das „severe acute respiratory syndrome coronavirus type 2" (SARS-CoV2) überhaupt die Pandemie verursachte. Die Autoren dieser Serie haben in ihrem Drehbuch Informationen verarbeitet, die schon lange vor COVID-19 bekannt waren. Denn die Krankheit „severe acute respiratory syndrome“ (SARS) ist nicht neu, sondern trat seit 2003 bereits in Kanada, China, Hongkong, Singapur, Vietnam und den Vereinigten Staaten auf (https://www.rki.de/DE/Content/ InfAZ/S/SARS/Klinik.html). Auch das Coronavirus ist nicht neu und löst in verschiedenen Varianten unterschiedliche Erkrankungen aus, von leichteren Erkältungskrankheiten bis zum „Middle East respiratory syndrome" (MERS) und eben SARS. Neu sind die derzeit grassierende Variante SARS-CoV-2 und die 
pandemische Verbreitung der COVID19.

Obwohl durchdachte und auf Langfristigkeit angelegte Pandemiepläne als Grundlage für politisches Handeln und Public-Health-Maßnahmen längst vorliegen, erstaunt das von Anfang an eher hilflos anmutende Agieren politisch Verantwortlicher in der Coronakrise. Das Robert Koch-Institut (RKI) erklärte zu seinem nationalen Pandemieplan bereits 2016:

Durch Standard-Hygienemaßnahmen, wie z. B. Händewaschen und Abstandhalten zu Erkrankten, kann das Risiko einer Atemwegsinfektion inklusive Influenza vermindert werden. Im Haushalt kann das Tragen eines Mund-Nasen-Schutzes in Kombination mit einer intensivierten Händehygiene bei allen Haushaltsmitgliedern die Übertragung von Influenza reduzieren - vorausgesetzt, dass diese Maßnahmen sehr früh nach Symptombeginn und von allen Mitgliedern im Haushalt (und den Erkrankten selbst) angewendet werden. ... Eine Impfung, die vor einer Ansteckung mit dem pandemischen Influenzavirus schützt und die wichtigste Präventionsmaßnahme darstellt, ist erst im Laufe der Pandemie verfügbar. (www.rki. de - Influenza-Pandemieplanung: Antworten auf häufig gestellte Fragen zur Pandemieplanung, Stand 05.12.2016)

Trotzdem wurde sowohl von den politischen Entscheidungsträgern (vermutlich auch angesichts der mangelnden Verfügbarkeit von Mund-Nasen-Schutz, MNS) als auch von Teilen der Öffentlichkeit und der Wissenschaft die Sinnhaftigkeit des Tragens eines MNS zu Beginn der SARSCoV-2-Pandemie monatelang vehement infrage gestellt. Erst eine im Juni veröffentlichte Metaanalyse von Chu et al. (2020) beendete zumindest in wissenschaftlichen Kreisen die Diskussion um die Sinnhaftigkeit des MNS-Tragens zur Ansteckungsprophylaxe, da in der Studie sowohl eine absolute Risikoreduktion durch Abstandhalten (von 12,8 auf 2,6\%) als auch durch MNS-Tragen (von 17,4 auf $3,1 \%$ ) überzeugend belegt wurde (zur Diskussion um die Wirksamkeit von MNS: Peeples 2020).

Die im Frühjahr 2020 beginnenden Spekulationen über einen schnell verfügbaren Impfstoff mit einem prognostizierten Ende der Pandemie für den Herbst 2020 wichen nach und nach der ernüchternden - wenngleich für Fachleute keineswegs überraschenden Erkenntnis, dass die Entwicklung und Verfügbarkeit eines Impfstoffes weit mehr Zeit benötigen als einige Monate. Der Verlauf der Pandemie wurde daher vom Autor der Heinsberg-Studie, Hendrik Streeck (https://www.uni-bonn.de/ neues/111-2020; Streeck et al. 2020), bildlich als „Marathon“ bezeichnet, als Warnung vor der Durchsetzung von auf „Sprint“ angelegten Schnellschussmaßnahmen.

Impfstoffe können angesichts der Unterschiedlichkeit von Viren erst entwickelt werden, wenn eine neue Virusvariante bereits aufgetreten ist. Die jahrzehntelangen Erfahrungen mit Impfstoffentwicklungen beschleunigen zwar diesen Prozess, sie zeigen aber auch, dass der Wunsch nach rascher Verfügbarkeit nicht zulasten der wissenschaftlichen Sorgfaltspflicht bei der Zulassung gehen darf. Es gibt aber dennoch Gemeinsamkeiten der meisten bisherigen Virus- und Bakterienepidemien und -pandemien, von der Pest (durch das Bakterium Yersinia pestis, meist übertragen von Flöhen und Ratten) über Pocken (Übertragung des Variolavirus von Mensch zu Mensch) bis zur sog. Spanischen Grippe, zu Erkältungskrankheiten und eben den neueren Varianten des SARS-CoV-2. Diese helfen, im Pandemiefall schnell und effektiv wirksame Schutzmaßnahmen der öffentlichen Gesundheitsvorsorge zu ergreifen. Diese Gemeinsamkeiten sind z. B.:

- oft Weitergabe von Mensch zu Mensch über Tröpfchen- bzw. Aerosolübertragung über die Mund- und Nasenschleimhäute,

- häufig Übertragung von auf engem Raum lebenden Tieren auf den Menschen und zurück, mit Tieren als Zwischenwirten während der Virusverbreitung,

- Übertragung über kontaminierte Oberflächen, die von vielen infizierten Personen angefasst werden, wie Türgriffe, Geländer usw., insbesondere, wenn diese aus eher porösen Materialien wie Kunststoff bestehen.
Allein aus diesen bekannten Fakten ergeben sich wahrscheinliche Verbreitungsszenarien, wie:

- bevorzugte Übertragung in der kalten Jahreszeit und in Innenräumen, wenn Menschen enger zusammen sind, Luftaustausch seltener stattfindet und die Schleimhäute trockener sind,

- besonderes Risiko in Tierfarmen, bei Massentierhaltung, auf Tiermärkten,

- hohes Übertragungsrisiko in öffentlichen Verkehrsmitteln, öffentlichen Gebäuden, bei Massenveranstaltungen, in Schulen und Kindergärten, in Großraumbüros, Krankenhäusern und Pflegeheimen.

Auf der anderen Seite ergeben sich daraus Ansätze für generelle Schutzmaßnahmen, wie:

- Tragen von MNS, um sowohl die Abgabe als auch die Aufnahme von infiziertem Tröpfchen- und Aerosolmaterial einzudämmen (mit einfachen Masken) oder zu verhindern (ab „Filtering-face piece“[FFP]Masken der Klasse 2), Händewaschen, Desinfektion von Oberflächen und Lüften,

- Verhinderung bzw. Einschränkung von Massenveranstaltungen, größeren Feiern usw.,

- Entzerrung von Gruppen in Kitas, Schulen, Universitäten, Arbeitsstätten, beim Einkaufen, in Gaststätten bzw. allgemein Abstand halten.

Aus der epidemiologischen Forschung ist bekannt, dass alle bislang erfolgreich bekämpften Infektionskrankheiten, die noch vor 100 Jahren den Großteil des Sterbegeschehens auch in den sog. Industrieländern ausmachten (wie Cholera, Typhus, Diphtherie, Scharlach, Tuberkulose, Masern), nicht durch einen Impfstoff zurückgedrängt wurden, sondern durch allgemeine Schutzmaßnahmen, wie verbesserte Hygiene, sauberes Trinkwasser, räumliche Trennung von Mensch und Nutztier, saubere und größere Wohnungen (Stroebe und Stroebe 1998). Um Missverständnisse zur vermeiden: Für diese Krankheiten wurden Impfstoffe entwickelt, die in vielen Fällen zu einer mindestens zeitweisen Eradikation beitrugen; zum Zeitpunkt der Ver- 
fügbarkeit der Impfstoffe war jedoch in allen Fällen der Zenit der Infektionsraten längst überschritten.

Die Zusammenfassung dieser bereits vor der COVID-19-Pandemie vorliegenden Evidenz und der während Frühjahr und Herbst 2020 gemachten Erfahrungen mit dem neuen SARS-CoV-2 ergibt für einen auf Weitsicht angelegten erfolgreichen Pandemieplan (vgl. RKI 2020) Die folgenden Eckpunkte: Diese werden auch in 2 Positionspapieren des Novembers 2020 (https://www. kbv.de/media/sp/KBV-Positionspapier_ Wissenschaft_Aerzteschaft_COVID-19. pdf und https://www.dgepi.de/assets/ Brief-Gem_Positionspapier_DurchDen Winter.pdf) von wissenschaftlichen Fachgesellschaften (u.a. der Deutschen Gesellschaften für Epidemiologie, Public Health, Sozial- und Präventivmedizin sowie Ärzte-, Psychotherapeuten- und Versorgungsverbänden) vertreten:

- Beobachtung des Pandemiegesche-

hens unter Berücksichtigung mög-

lichst vieler Parameter; neben der

Zahl der Neuinfektionen (Inzidenz)

sollten auch Infektionszahlen (Präva-

lenz), Zahl durchgeführter Tests, einschließlich Art der Tests (z. B.

Antigenschnelltests vs. Polymerase-

Kettenreaktion[PCR]-Tests), sta-

tionäre und intensivmedizinische

Behandlungskapazitäten - nicht nur materiell, sondern auch personell in einem möglichst einheitlichen und leicht zu verstehenden Bewertungssystem (z. B. Ampelsystem) berücksichtigt und öffentlich kommuniziert werden.

- Ein langfristig achtsamerer Umgang der Menschen untereinander zum Selbst- und Fremdschutz muss etabliert werden (Abstand halten, Hygieneregeln, insbesondere im öffentlichen Raum, beachten, in geschlossenen Räumen und insbesondere in der kalten Jahreszeit MNS beim Zusammentreffen mit anderen außerhalb der häuslichen Umgebung tragen, regelmäßig Lüften, „kontextsensible Kontaktreduktion“). Dies sollte, wo immer möglich, über Gebote statt Verbote realisiert werden, da Verbote Reaktanz provozieren und ohnehin nur wirksam sein kön-

Psychotherapeut 2021 ·66:186-194 https://doi.org/10.1007/s00278-021-00505-6

(c) Springer Medizin Verlag GmbH, ein Teil von Springer Nature 2021

\section{B. Strauß ·. Rosendahl · U. Berger}

\section{Bedeutung der COVID-19-Pandemie für die öffentliche Gesundheit und gruppenpsychologische Aspekte - Teil 2 einer (vorläufigen) Übersicht}

\section{Zusammenfassung}

Aufbauend auf den Befunden zu Auswirkungen der durch die "coronavirus disease 2019" (COVID-19) ausgelösten Pandemie auf die Lebensbedingungen und die seelische Gesundheit von Menschen im ersten Teil der Übersicht gehen die Autoren im Folgenden auf die Konsequenzen der Pandemie für die öffentliche Gesundheit ein. Für den Public-Health-Aspekt von COVID-19 ist verblüffend zu sehen, dass - im Übrigen auch bezüglich psychologischer Faktoren und Konsequenzen der Pandemie - ein fundiertes Wissen, basierend auf früheren Pandemien und Katastrophen, eigentlich schon lange vor dem COVID-19-Ausbruch bereitstand, dass dieses Wissen aber, wenn überhaupt, erst zögerlich für gesundheitspolitische Entscheidungen genutzt wurde. Angesichts der sozialen Bedeutung der Pandemie und deren sozialen Auswirkungen kommt Befunden der Gruppenpsychologie und -dynamik sowohl für das Verständnis von Verhaltensweisen in der Bevölkerung wie auch für die Planung von Maßnahmen der öffentlichen Gesundheit bezüglich der Pandemie eine große Bedeutung zu. Im Kontext der Pandemie wurde eine Zunahme spezifischer psychischer Störungen beschrieben, für deren Behandlung potenziell auch evidenzbasierte gruppentherapeutische Angebote zur Verfügung stehen. Während der Einsatz von telemedizinischen und digitalen Techniken in der Einzeltherapie mittlerweile schon relativ verbreitet ist, sind in diesem Kontext bezüglich Gruppentherapien noch viele Fragen offen.

Schlüsselwörter

Public Health · Prävention · Gruppendynamik . Gruppentherapie - Gesundheitswissen, -einstellungen, -praxis

\section{Importance of the COVID-19 pandemic for public health and group psychological aspects-Part 2 of a (preliminary) review}

\section{Abstract}

Based upon the findings related to the impact of the coronavirus disease 2019 (COVID-19) pandemic on human living conditions and psychological health, in the first part of this review the authors discuss the consequences of the pandemic for public health. It is surprising to see that related to public health but also psychological factors and sequelae of the pandemic, a broad knowledge was already available based upon former pandemics and disasters long before the outbreak of COVID-19. This knowledge has been used very sparsely, if at all, for health political decisions. In view of the social significance of the pandemic and its social impact, findings from group psychology and group dynamics seem to be specifically important for a better understanding of behavior within the population as well as the conceptualization of public health interventions. An increase in psychological disorders was described related to the pandemic. For the treatment of these disorders, a range of psychotherapeutic approaches including evidence-based group psychotherapy are available. Whereas the use of telemedical and digital techniques is increasingly more common within individual psychotherapy, many questions are still open related to online group treatment.

Keywords Public health · Prevention - Group dynamics . Group psychotherapy · Health knowledge, attitudes, practice nen, wenn sie flächendeckend mit Kontrollen und Strafen unterlegt werden.

- Besonderer Schutz von Risikogruppen (Ältere, Personen mit Vorerkrankungen usw., siehe die Taxonomie im ersten Teil der Übersicht (Strauß et al. 2021)) und Personal in Krankhäusern, Pflegeheimen, aber auch im öffentlichen Verkehr, in Ladengeschäften und allgemein bei verstärktem Publikumsverkehr und Kontakt mit Personen außerhalb des eigenen Haushalts. 
Hier steht eine Anzeige.

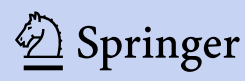


- Schaffung von Hygienekonzepten für jede Art von Zusammentreffen mehrerer Personen, d. h. bei Veranstaltungen, in der Gastronomie, in Bildungseinrichtungen, in Arbeitsstätten usw., aber keine generellen Schließungen (Lockdown), solange die Kapazitätsgrenzen im Gesundheitssystem noch nicht erreicht sind.

- Bessere und breitere Nutzung der Corona-App und zusätzliche Schaffung Smartphone-unabhängiger Lösungen.

- Sowohl die Öffentlichkeit als auch alle Personen, die mit der Durchführung von Tests auf eine mögliche Infektion mit SARS-CoV-2 betraut werden, müssen besser über die Aussagekraft solcher Tests aufgeklärt werden; dazu müssen gut aufbereitete und einfach verständliche Informationen deutlich mehr verbreitet und zugänglich gemacht werden, als dies bislang der Fall ist.

- Transparenz und Planbarkeit von Zeitpunkt und Ausstieg bei verschärften Maßnahmen sowie Bildung eines gesellschaftlichen Konsenses bei Priorisierung bestimmter Gruppen bzw. Institutionen (wie Kitas und Schulen, Gaststätten- und Beherbergungsbetriebe, kulturelle Veranstaltungen).

Diese Punkte stehen z.T. im Widerspruch zu den von der Bundesregierung (und auch den Regierungen in anderen Ländern) veranlassten Maßnahmen. Auffällig ist insbesondere das kurzfristige und reaktive Agieren. So wurde z.B. der Sommer mit einem kompletten Absinken der Infektionsraten nicht genutzt, um Personal in den Kliniken aufzustocken und den Präsenzunterricht in den Bildungseinrichtungen (v.a. in den Schulen) schwerpunktmäßig in diesen Zeitraum zu verlegen (durch Kürzung der Sommerferien und Verlängerung der Winterferien). Ein Lockdown der Gastronomie, wie er für den November 2020 bundesweit angeordnet wurde, ist absehbar nicht mehrfach finanzierbar bzw. würde zu massenhaften Insolvenzen im Gaststätten- und Hotelgewerbe führen. Umgekehrt wäre eine Aufstockung des Pflegepersonals in den Kliniken (v.a. im Intensivpflegebereich) eine Investition in die Zukunft, da der sog. Pflegenotstand bereits vor der COVID-19-Pandemie bestand und gleichzeitig ein Nadelöhr bei der Pandemiebekämpfung ist (Böhmer 2020; https://doi.org/https://www.aerzteblatt. de/nachrichten/sw/pflegenotstand?s=\& $\mathrm{p}=1 \& \mathrm{n}=1 \& \mathrm{nid}=118950)$. Zudem sind in dem am 18.11.2020 von Bundestag und Bundesrat verabschiedeten neuen Pandemiegesetz ( $\$ 28 \mathrm{a}$ Infektionsschutzgesetz, 3. Bevölkerungsschutzgesetz, https://www.bundestag. de/dokumente/textarchiv/2020/kw47de-bevoelkerungsschutz-804202) zwar konkrete Maßnahmen benannt (die jedoch fast ausnahmslos den bisher bereits durchgeführten Maßnahmen entsprechen), aber kaum Ein- und Ausstiegskriterien definieren.

\section{Gruppenpsychologie im Kontext der Pandemie}

In einem ungewöhnlichen Beitrag für den Guardian (vom 17.05.2020, Fonagy 2020) vermittelte der eigentlich mehr in der Psychotherapieforschung bekannte Peter Fonagy die Botschaft, dass in diesen Zeiten Freundlichkeit und soziale Aufgeschlossenheit insbesondere gegenüber vulnerablen Personen von außergewöhnlicher Bedeutung seien. Dieser Appell ist eine von vielen Botschaften, die die soziale und Gruppendimension der COVID-19-Pandemie tangieren, wobei zu Recht auf die Zweischneidigkeit der Gruppenperspektive in diesem Kontext verwiesen wurde (z. B. Marmarosh et al. 2020): Einerseits gelten seit Monaten körperliche Nähe und die $\mathrm{Zu}$ sammenkunft von Gruppen als Gefahr und Ursache für die Verbreitung der Erkrankung, auf der anderen Seite sind Gruppenzusammenkünfte bekanntermaßen auch ein probates Mittel gegen Stress und Belastungen: „An intervention intended to reduce the likelihood of physiological illness likely contributes to a rise in likelihood of psychological illness" (Marmarosh et al. 2020, S. 124).

Auch im Gruppenkontext wurde die Frage gestellt, ob Gruppenforscher in Interventionsteams im Bereich der öffentlichen Gesundheit viel zu sehr unterre- präsentiert waren/sind, zum einen, da diese auf die Notwendigkeit von Gruppenerfahrungen hinweisen würden, zum anderen aber auch, weil die Tendenz zu Verstößen gegen die öffentlichen Pandemieregeln gruppendynamisch durchaus vorhersagbar wäre.

In einem Beitrag aus einem Themenheft der Zeitschrift Group Dynamics mit dem Titel „Groups in a dangerous time: virtual work in therapy in the COVID19 era" beschreibt Forsyth (2020) das alte Konzept des Gruppendenkens („groupthink") mit Perspektive auf die Pandemie: Dieses Konzept stammt aus den frühen 1970er-Jahren (Janis 1972) und stellt den Versuch dar, schlechte Gruppenentscheidungen zu erklären. Ein Ausgangspunkt war damals die Entscheidung einer Beratergruppe John F. Kennedys, die Schweinebucht auf Kuba zu besetzen. Janis (1972) fand im Zusammenhang mit diesem Ereignis, dass in diesem Kontext ein spezifisches Gruppendenken (mit negativen Folgen) wirksam war, für das er eine ganze Reihe von Faktoren verantwortlich machte, die auch in Zeiten von Corona allenthalben beobachtbar sind: Zum einen gehört zum Gruppendenken der Einfluss eines charismatischen Gruppenleiters mit einer bekannten Agenda (damals John F. Kennedy, bis vor kurzem in den USA beispielsweise Donald Trump, hierzulande z.B. die Wortführer, die sich aufCoronademonstrationen zeigen). Die betroffene Gruppe verweigert jeglichen Input von außerhalb, bzw. dieser Input wird nicht wahrgenommen, da die Gruppengrenzen nicht mehr durchlässig sind. Wenn innerhalb der Gruppe nicht widersprochen wird, gilt dies als Zeichen von Zustimmung (ebenso das Schweigen). Betroffene Gruppen haben eine hohe Kohäsion im Angesicht einer großen Belastung bzw. einer potenziell bedeutenden Entscheidung. Dies führt zusammengenommen zu einer hohen sozialen und ideologischen Homogenität der Mitglieder. Gruppendenken wird darüber hinaus gefördert, wenn in der Gruppe kein geregeltes Entscheidungsverfahren existiert.

Wie erwähnt, wurde von Janis (1972) u. a. das Konzept Groupthink im Kontext schlechter politischer Entscheidungen, Entscheidungen an der Börse oder 


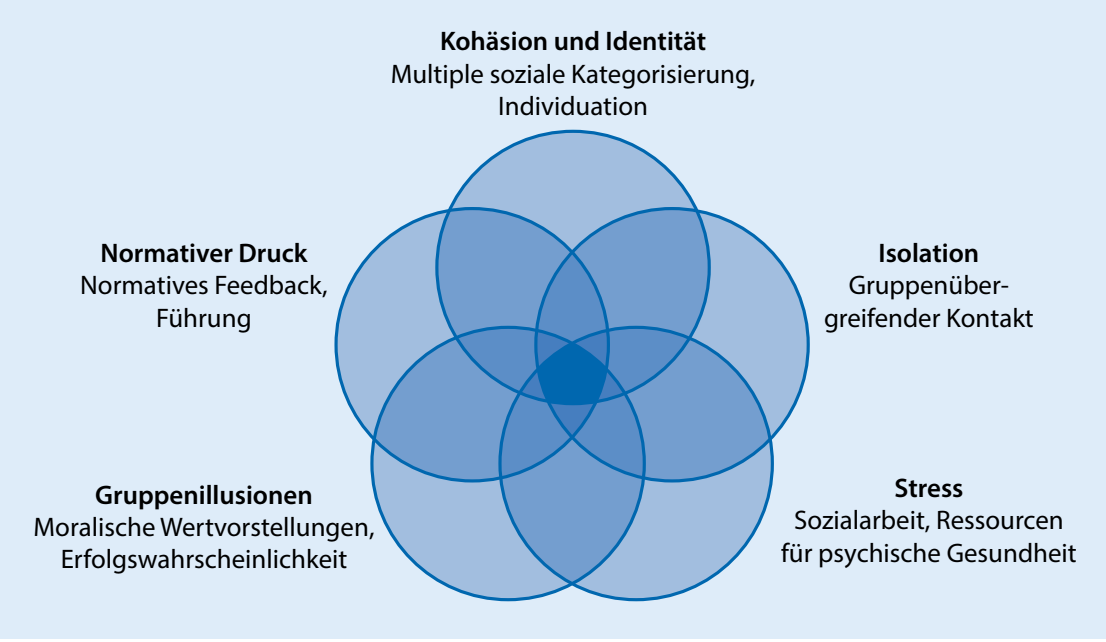

Abb. 1 Matrix möglicher Interventionen auf Gruppenebene zur Minimierung von Widerstand gegen Gesundheitsinitiativen. (Nach Forsyth 2020)

beispielsweise auch im Hinblick auf die fehlgeschlagene Operation der National Aeronautics and Space Administration (NASA), trotz widriger meteorologischer Bedingungen die Raumfähre Challenger starten zu lassen, entwickelt. Forsyth (2020) betrachtet das Konzept aus heutiger Sicht und kann zeigen, dass auch unter dem Einfluss zusätzlicher Faktoren Gruppendenken viele negative Reaktionen auf die Maßnahmen gegen die COVID-19-Pandemie bestimmt. Diese zusätzlichen Faktoren sind möglicherweise Isolation (etwa infolge von Quarantäne; Brooks et al.2020) und verschiedene „Gruppenillusionen“, wie die Illusion der Moralität, der Unverletzlichkeit und der Einstimmigkeit sowie ein ausgeprägter normativer Druck.

Während Gruppendenken ursprünglich in Gruppen für besonders wahrscheinlich gehalten wurde, die bereits über eine Geschichte guter Entscheidungen verfügen und in denen sich eher talentierte und intelligente Mitglieder befinden, kann man nach den heutigen Erkenntnissen davon ausgehen, dass Gruppendenken auch in geschichtslosen Ad-hoc-Gruppen möglich wird, wobei diese durch „eine willige Ignoranz gekennzeichnet" sind. Dies bedeutet, dass Gruppen, die nach diesem Modell funktionieren, sich genau zu ihren Einstellungen passende Informationen suchen, auch wenn diese offenkundig falsch sind, was gerade im COVID-19-Umfeld ver- mutlich nicht schwierig ist. Eine Matrix für mögliche Interventionen auf Gruppenebene, mit denen beispielsweise der Widerstand gegen Gesundheitsinitiativen (und damit das Gruppendenken) verändert werden kann, zeigt • Abb. 1 (in Anlehnung an Forsyth 2020).

Die Matrix umfasst 5 Bereiche, nämlich die bereits erwähnten Gruppenillusionen, den normativen Druck, der naturgemäß durch Autoritätspersonen innerhalb und außerhalb einer Gruppe beeinflusst werden kann (John F. Kennedy beispielsweise soll nach der Invasion der Schweinebucht ganz gezielt seine Beratergruppe aufgefordert haben, Widerspruch zu dulden, Isolation $\mathrm{zu}$ verringern und warnte vor vorschnellen Schlüssen, während etwa Donald Trump über seine Tweets den normativen Druck, gegen gesundheitsbezogene Regeln zu verstoßen, massiv aufbaut und damit den Widerstand gegen Quarantäne und sonstige Präventionsmaßnahmen erhöht). Weitere Faktoren sind die Isolation, die man über den Kontakt zu anderen Gruppen regulieren könnte, die Differenzierung sozialer Identität und von Kohäsion sowie das Ausmaß an Stress, das beispielsweise durch entsprechende Angebote der Stressreduktion modifiziert werden kann.

Die gruppendynamische Literatur (Übersicht bei Forsyth 2020) gibt also durchaus Hinweise für präventive Aspekte, u. a. Ergebnisse aus der Kon- formitätsforschung, wo man ablesen kann, dass Gruppen mit einem hohen $\mathrm{Ma} ß$ an Skepsis gegenüber einer Krise sehr viel deutlicher dazu beitragen, dass kritische Stimmen, die eine Gefahr realistisch einschätzen, unberücksichtigt bleiben. Es kann auch gezeigt werden, dass Einzelpersonen sich in ihrem Urteil oft viel mehr durch die Einstellungen Nahestehender beeinflussen lassen als durch objektives Wissen, dass allerdings umgekehrt aber eine metakognitive Informationsverarbeitung deutlich mehr auf repräsentatives Wissen, denn auf anekdotische Fälle zurückgeführt wird (Stasser und Abele 2020). Schon Hill (1987) hat gezeigt, dass eine Vernachlässigung von Forderungen nach sozialer Distanz mit dispositionellen Unterschieden im Hinblick auf die Präferenz erklärt werden kann, im Fall von Stress andere Menschen zu kontaktieren (Forsyth 2020).

Eine ganze Reihe von Faktoren wird in der Literatur beschrieben, die auf individueller und auch Gruppenebene Reaktionen auf Interventionen gegen die Verbreitung der Pandemie negativ beeinflussen. Auf einer individuellen Ebene sind es offensichtlich vielfältige Faktoren, wozu etwa ein erhöhtes Maß an Narzissmus und Psychopathie gehört; in jüngster Zeit wird in diesem Kontext auch die „dunkle Triade“ untersucht (Nowa et al. 2020). Auf einer Gruppenebene sind es insbesondere unterschiedliche Arten von normativem Druck und das Gruppendenken, die das Verhalten der Gruppe und des Einzelnen bestimmen.

\section{Telemedizinische grup- penpsychotherapeutische Behandlungsansätze?}

\section{Ein Schub für die Telepsycho- therapie}

Im Einzeltherapiebereich ist mittlerweile Teletherapie als hoch wirksam beschrieben (z.B. Andersson 2018). "Remote formats" (Telefon, Internet) scheinen beispielsweise gut belegte Formate für kognitiv-behaviorale Therapie (CBT) und interpersonale Psychotherapie (IPT) bei (postnatalen) Depressionen zu sein (z. B. Dennis et al. 2020). So ist es 
nicht erstaunlich, dass in unserem Gesundheitssystem infolge der Pandemie und des Lockdowns viele psychotherapeutische Kontakte im individuellen Bereich auf Videosprechstunden und telemedizinische Formate verlagert wurden, wenngleich auch mit einer gewissen Ambivalenz und Selbstzweifeln aufseiten der Psychotherapeut ${ }^{*}$ innen (Aafies-van Doorn et al. 2020) sowie kritischen Stimmen zu den möglichen Einschränkungen aus technischer, aber auch aus sozialer, psychotherapeutischer und ethischer Perspektive (Tullio et al. 2020).

Tatsächlich scheint sich - nicht zuletzt aufgrund der Angst der Psychotherapeut ${ }^{*}$ innen vor Ansteckung - eine Reduktion realer zugunsten von telefonischen und Internetkontakten als Folge von COVID-19 ergeben zu haben (z. B. Humer et al. 2020; Inchausti et al. 2020), was auch als eine positive Wirkung der Pandemie gewertet wird (Torous und Wykes 2020; Wright und Caudill 2020). Eine ausführlichere Übersicht hierzu bietet der Beitrag von Eichenberg (2021, in diesem Heft).

Die Anwendung gruppenbezogener Interventionen via Internet ist vergleichsweise noch deutlich weniger verbreitet und erprobt. Angesichts der Bedeutung gruppenpsychologischer Betrachtungen der Pandemie und ihrer Auswirkungen überrascht es nicht, dass auch bezüglich (neuer) Gruppenformate im klinischtherapeutischen Bereich infolge der COVID-19-Pandemie diskutiert wird.

\section{Gruppenpsychotherapie in Zeiten von COVID-19}

Wie in Teil I der Übersicht gezeigt (Strauß et al. 2021), ist eine Vielzahl psychischer Probleme als Folge der Pandemie in der noch jungen epidemiologischen Literatur beschrieben, allen voran Angststörungen, affektive Störungen, belastungsabhängige Störungen, aber auch der erhöhte Missbrauch von Substanzen, wie Drogen und Alkohol. Die gruppenpsychotherapeutische Literatur (beispielsweise die aktuelle Zusammenfassung von Strauß et al. 2020) Weist aus, dass mittlerweile für eine Vielzahl eben dieser, aber auch anderer psychischer Störungen eine sehr überzeugende Evidenz zur Wirksamkeit von Gruppentherapien vorliegt. Diese Wirksamkeit lässt sich sowohl im Vergleich mit "inaktiven“ Kontrollgruppen, wie Wartelisten etc., nachweisen, aber auch im Vergleich zu anderen aktiven Behandlungsmaßnahmen (einschließlich der Einzeltherapie), bei denen sich keine bedeutsamen Unterschiede zwischen Gruppen und anderen Behandlungssettings finden lassen (z. B. Burlingame et al. 2016).

$\mathrm{Da}$ auch aus hygienischen Gründen Live-Gruppen in Zeiten von COVID-19 schwierig geworden sind, stellt sich die Frage, ob es digitale oder virtuelle Alternativen gibt, die man auch für die Gruppenbehandlung in Zeiten von COVID19 nutzen kann, nachdem viele psychotherapeutische Kontakte im individuellen Bereich ja auf Videosprechstunden und telemedizinische Formate verlagert wurden.

Im Gruppenbereich gibt es schon lange verschiedene Formen von „Onlinegruppen", angefangen von E-Mail-Foren, in denen man die anderen Gruppenmitglieder nicht zu Gesicht bekommt, und asynchrone Gruppen, die sich eines Internetforums bedienen, in das man sich nach Bedarf einwählen kann und in dem man in erster Linie Meinungen und Informationen aufgreift. In Zeiten von COVID-19 sind synchrone Gruppen bedeutsamer geworden, vor allen Dingen solche, die mit Videounterstützung arbeiten, wie etwa Zoom-basierte Gruppen (Armington et al. 2020). Bislang sind die Forschung und auch das Ausmaß an Erfahrung bezüglich Onlinegruppenpsychotherapien insbesondere im nicht verhaltenstherapeutischen Bereich nicht sehr ausgeprägt. Innerhalb der ambulanten psychotherapeutischen Struktur herrscht momentan offenbar noch eher eine ablehnende Haltung gegenüber Telegruppentherapien, zumindest hierzulande, während in anderen Ländern seit dem Ausbruch der COVID-19Pandemie doch mehr Erfahrungen vorliegen, da die alternative Einhaltung von strengen Hygienemaßnahmen wichtiges Einkommen reduziert hätte. Möglicherweise ist beispielsweise in den USA auch das Bewusstsein für den Datenschutz ebenso anders wie die Forderung von Patienten, durch teletherapeutische
Angebote große Entfernungen nicht mit Verkehrsmitteln überwinden zu müssen.

Ein Autor, der sich sehr intensiv mit der Frage der Gruppenteletherapie beschäftigt hat, ist Haim Weinberg (2020a), der in dem Buch Theory and practice of online therapy (Weinberg und Rolbnick 2019) diskutiert, welchen Problemen man in diesem Kontext potenziell begegnen kann. Er weist auf die Aspekte von Vertraulichkeit und Datenschutz, auf die Frage, welche Mitglieder letztlich bzw. am ehesten für eine Gruppenteletherapie ausgewählt werden sollten, auf die Tatsache, dass die Onlinegruppe eine körperlose Gruppe ist, ebenso wie auf die Frage der tatsächlichen Präsenz und der Kontrolle über das Setting, das natürlich nicht mehr gänzlich in der Hand des Gruppenleiters liegt, hin.

Weinberg (2020b) geht auch auf die Frage des Hintergrunds ein und kommentiert:

If someone brought a cat to the group, most group leaders would explore the $d y$ namic meaning for the participant who brought the cat. However, when someone passes behind one of the group members when they sit in front of a computer, or when the tail of a cat suddenly appears on the screen, most of the time no one, including the group therapist, comments on it. It is as if these background details become transparent to us. Special attention and training are needed not to ignore these events. (Weinberg 2020b)

Weinberg (2020a) resümiert, dass die Onlinegruppentherapieforschung noch in ihren Kinderschuhen steckt und wir vor allen Dingen viel zu wenig über die Effektivität von Gruppenangeboten für einzelne Mitglieder wissen. Im Großen und Ganzen gibt es keine randomisierten kontrollierten Studien zur Wirksamkeit von Videogruppen im Vergleich zu anderen Ansätzen.

In Weinbergs Sichtweise können zwei wesentliche Aspekte der therapeutischen Allianz, nämlich die Übereinstimmung bezüglich der Therapieziele ("goals") und der Frage ihrer Zielerreichung (,task“) in Onlinegruppen durchaus erreicht werden, wohingegen die Frage der emotionalen Qualität der Beziehung („bonds“) noch nicht geklärt ist. Diese dürfte in star- 
kem Maße mit körperlicher Interaktion einschließlich Augenkontakt in Verbindung stehen, der in Videogruppensettings nicht bzw. sehr viel schwerer herstellbar ist. Darüber hinaus ist zu vermuten, dass wirkliche Präsenz über Bildschirmkontakte u.a. auch aufgrund zu vieler Ablenkungsfaktoren sehr schwer herstellbar sein wird. Hier ist also sehr viel Forschung nötig, wobei auch einige Hinweise aus der gruppendynamischen Forschung im nichtklinischen Bereich wichtig sein können. So konnten z.B. Holtz et al. (2020) zeigen, dass a priori erteilte Instruktionen und Hinweise auf den Umgang mit Emotionen interaktive Probleme zumindest in nichtklinischen virtuellen Gruppen deutlich reduzieren können und dazu beitragen, dass virtuelle und nichtvirtuelle Gruppen durchaus ähnlich wahrgenommen werden (Brown et al. 2020), wobei der Umgang mit virtuellen Gruppen interindividuell stärker unterschiedlich ist. Beispielsweise ist die Bereitschaft zur Nutzung abhängig von der technischen Ausstattung, den Fertigkeiten im Umgang mit Technologie und den ganz allgemeinen Einstellungen zur Technologie sowie von der Nutzung der hierfür zur Verfügung gestellten „Tools“ im Sinne der Entwicklung von Standards, mit denen alle gleichermaßen gut umgehen können.

Insgesamt gesehen kommen Gruppendynamiker aber zu dem Schluss, dass Gruppen im Angesicht der aktuellen Pandemie einen gewaltigen Aufschwung erleben sollten:

The light is shining more brightly than ever on the power of group psychotherapy to help people quickly make meaningful improvements in their quality of life. Hopefully, all of this will spur interest in groups, interest in funding scholars of groups, and willingness to more fully underwrite group-based treatments for mental and life issues (Parks 2020, S. 120).

\section{Ausblick/Diskussion}

Einige Aspekte, die in dieser Übersicht gezeigt wurden, legen nahe, dass die momentan wie am Fließband produzierten Befunde zu psychosozialen Aspekten der COVID-19-Pandemie wis- senschaftliche Erkenntnisse bestätigen, die bereits vor dem Ausbruch prinzipiell bekannt waren. Hierzu zählen die potenzielle Steigerung einer Vielzahl psychischer Probleme und Störungen, die Notwendigkeit spezifischer Angebote, auch psychotherapeutischer Art, für die Betroffenen und die Betrachtung der gruppendynamischen Prozesse rund um die Pandemie, einschließlich der Reaktionen bestimmter Teile der Bevölkerung. So gesehen dürften die Inhalte der Übersicht vielleicht doch nicht so schnell veraltet sein (Strauß et al., in diesem Heft; Spitzer 2020). Zusammenfassend sind auch aus der Public-Health-Perspektive COVID-19 und SARS-CoV-2 vom grundsätzlichen Agieren her in Bezug auf den Bevölkerungsschutz keine einmalige oder neue Herausforderung. Wenngleich dies 100 Jahre nach der sog. Spanischen Grippe die erste Infektionskrankheit wirklich pandemischen Ausmaßes ist, wird es erwartungsgemäß weder die Letzte sein, noch gibt es eine Garantie dafür, dass bis zur nächsten Pandemie wieder 100 Jahre vergehen werden (insofern entspricht die Bezeichnung "Jahrhundertpandemie“ vom Gesundheitsminister Jens Spahn im Bundestag am 18.11.2020 wohl eher dessen Wunschdenken). Die in den vergangenen Jahrzehnten sprunghaft gestiegene globale Mobilität, die Zunahme von Massentierhaltung, das weltweite Bevölkerungswachstum und die globale Erwärmung im Zuge des Klimawandels sprechen für zunehmend bessere Entstehungs-, Überlebens- und Verbreitungschancen insbesondere von Viren, die Menschen und Tiere wechselseitig als Wirte nutzen und die eine hohe Mutationsrate mit immer neuen Varianten aufweisen. Insofern wäre es gut, wenn die Gesundheitspolitik nicht nur aus den Erfahrungen mit dem COVID19-Virus, sondern auch mit früheren Pandemien und den damit verbundenen Erkenntnissen aus einer psychosozialen und Public-Health-Perspektive lernen könnte und das sozialwissenschaftliche Wissen und die Kompetenz in diesen Feldern auch noch adäquater für die Entscheidungsfindung auf dem Feld der Gesundheitspolitik und der öffentlichen Gesundheit nutzen würde.

\section{Fazit für die Praxis}

- Die durch die „coronavirus disease 2019“ (COVID-19) ausgelöste Pandemie stellt die öffentliche Gesundheit vor Probleme, die eigentlich bereits antizipiert waren. Entsprechend gibt es eine ganze Reihe von Empfehlungen und Plänen, wie mit Pandemien umzugehen ist.

- Zum Verständnis der Reaktionen auf die Pandemie, aber auch des Umgangs mit Maßnahmen der öffentlichen Gesundheit eignen sich Konzepte aus der Gruppenpsychologie, wie etwa das Konstrukt des Gruppendenkens.

- Gruppenpsychotherapeutische Interventionen stehen für zahlreiche Probleme, die infolge der Pandemie vermehrt zu beobachten sind, zur Verfügung.

- Die Pandemie hat auch zu einem vermehrten Angebot an telemedizinischen Gruppentherapien geführt, deren differenzielle Wirkung aber noch weiter untersucht werden muss.

\section{Korrespondenzadresse}

Prof. Dr. Bernhard Strauß

Institut für Psychosoziale Medizin, Psychotherapie und Psychoonkologie, Universitätsklinikum Jena

Stoystr. 3, 07740 Jena, Deutschland

Bernhard.Strauss@med.uni-jena.de

\section{Einhaltung ethischer Richtlinien}

Interessenkonflikt. B. Strauß, J. Rosendahl und U. Berger geben an, dass kein Interessenkonflikt besteht.

Für diesen Beitrag wurden von den Autoren keine Studien an Menschen oder Tieren durchgeführt. Für die aufgeführten Studien gelten die jeweils dort angegebenen ethischen Richtlinien.

\section{Literatur}

Aafies-van Doorn K, Bekes V, Prout T (2020) Grappling with our therapeutic relationship and professional self-doubt during COVID-19. Couns Psychol Quart. https://doi.org/10.1080/09515070.2020. 1773404

Andersson G (2018) Internet interventions: past, present and future. Internet Interv 12:181-188

Armington R, Badenoch B, Gantt S (2020) Zooming along in the pandemic and beyond. https:// 
www.agpa.org/docs/default-source/practiceresources/article_zooming-along-in-thepandemic-and-beyond.pdf?sfvrsn $=41999 \mathrm{ba} 9$ 0.Zugegriffen: 13. März 2021

Böhmer N (2020) „Euren Applaus könnt ihr euch sonst wohin stecken" - Pflegenotstand, Materialmangel, Zeitnot - was alles in unserem Gesundheitssystem schiefläuft. HarperCollins, Hamburg

Brooks SK, Webster RK, Smith LEetal (2020) The psychological impact of quarantine and how to reduce it: rapid review of the evidence. Lancet. https:// doi.org/10.1016/S0140-6736(20)30460-8

Brown MI, Prewett MS, Grossenbacher MA (2020) Distancing ourselves from geographic dispersion. Group Dyn 24:168-185

Burlingame GM, Seebeck JD, Janis RA et al (2016) Outcome differences between individual and group formats. Psychotherapy 53:446-461

Chu DK, Akl EA, Duda S, Solo K, Yaacoub S, Schünemann HJ et al (2020) Physical distancing, face-masks, and eye protection to prevent person-to-person transmission of SARS-CoV-2 and COVID-19: a systematic review and metaanalysis.Lancet 395:1973-1987.https://doi.org/ 10.1016/S0140-6736(20)31142-9

Dennis CL, Grigoriadis S, Zupancic J et al (2020) Telephone-based nurse-delivered interpersonal psychotherapy for postpartum depression: nationwide randomised controlled trial. $\mathrm{Br}$ Psychiatry 216(4):189-196

Eichenberg C (2021) Online-Psychotherapie in Zeiten der Corona-Pandemie. Psychotherapeut. https://doi.org/10.1007/s00278-020-00484-0

Fonagy P (2020). Kindness can work wonders. Especially for the vulnerable. The Guardian, Sun 17 May 2020

Forsyth DR (2020) Group-level resistance to health mandates during the COVID-19 pandemic: a groupthinkapproach. Group Dyn 24:139-152

Hill CA (1987) Afficiliation motivation: people who need people ....but in different ways. J Pers Soc Psychol 52:1008-1018

Holtz K, Castella VO, Abad AZ, Gonzalez-Anta B (2020) Virtual team functioning. Modelling the affective and cognitive effects of an emotional management intervention. Group Dyn 24:153-167

Humer E, Pieh C, Kuska M (2020) Provision of psychotherrapy during the COVID.19 pandemic among Czech, German and Austrian Psychotherapists. Int JEnviron Res Publih Health 17:4811

Inchausti F, MacBeth A, Hasson-Ohayon I, Dimaggio G (2020) Psychological intervention and COVID19: what we know so far and what we can do. J Contemp Psychother. https://doi.org/10.1007/ s10879-020-09460-w

Janis IL (1972) Victims of groupthink. HoughtonMifflin, Boston

Marmarosh C, Forsyth D, Strauss B, Burlingame GM (2020) The psychology of the COVID.19 pandemic. A group-level perspective. Group Dyn 24:122-138

Nowa B, Brzóska P, Piotrowski J et al (2020) Adaptive and maladaptive behavior during the COVID19 pandemic: the roles of dark triad traits, collective narcissism, and health beliefs. Pers Individ Dif 167:110232.https://doi.org/10.1016/ j.paid.2020.110232

Parks CD (2020) Group dynamics when battling the pandemic. Group Dyn 24:115-121

Peeples L (2020) What the data say about wearing facemasks. Nature 586:186-189
Robert-Koch-Institut (RKI] Nationaler Pandemieplan Teil I und II. https://www.rki.de. Zugegriffen: 18 Nov. 2020

Spitzer M (2020) Psychologie und Pandemie. Nervenheilkunde 39(05):274-283

Stasser G, Abele S (2020) Collective choice, collabroation and communication. Annu Rev Psychol 71:589-612

Strauß B, Burlingame GM, Rosendahl J (2020) Neuere Entwicklungen in der Gruppenpsychotherapieforschung - ein Update. Psychotherapeut 65:225-235

Strauß B, Berger U, Rosendahl J (2021) Mittelbare und unmittelbare psychosoziale Folgen der COVID19-Pandemie - Eine (vorläufige) Übersicht: Teil I: Folgen für die psychische Gesundheit und Konsequenzen für die Psychotherapie. Psychotherapeut. https://doi.org/10.1007/s00278021-00504-7

Streeck H et al (2020) Infection fatality rate of SARSCoV-2 infection in a German community with a super-spreading event. medRxiv. https://doi. org $/ 10.1101 / 2020.05 .04 .20090076$

Stroebe W, Stroebe MS (1998) Lehrbuch der Gesundheitspsychologie - Ein sozialpsychologischer Ansatz. Dietmar Klotz, Frankfurt

Taylor S (2020) Die Pandemie als psychologische Herausforderung. Psychosozial-Verlag, Gießen

Torous J, Wykes T (2020) Opportunities from the coronavirus disease 2019 pandemic for transforming psychiatric care with telehealth. JAMA Psychiatry 77(12):1205-1206

Tullio V, Perrone G, Bilotta C et al (2020) Psychological support and psychotherapy via digital devices in COVID-19 emergency time: some critical issues. Med Leg J 88:73-76

Weinberg H (2020b) Form the circle to the screen. https://groupanalyticsociety.co.uk/webinarconductinjg-group-therapy-online. Zugegriffen: 13. März 2021

Weinberg H (2020a) Online group psychotherapy: Challenges and possibilities during COVID19-A practice review. Group Dyn 24:201-211

Weinberg H, Rolbnick A (Hrsg) (2019) Theory and practice of online therapy. Routledge, New York

Wright JH, Caudill R (2020) Remote treatment delivery in response to the COVID-19 pandemic. Psychother Psychosom 89:130-132

\section{Neuer Indikator zur Voraussa- ge von Stressresilienz}

Forschende der Universität Zürich zeigten mit einem realen Stressreiz auf, dass die erhöhte Aktivität eines bestimmten Hirnareals die Entstehung von Angst- und Depressionsstörungen begünstigt. Ihre Studie ist die erste, die ein objektives, neurobiologisches Mass beschreibt, das die Stressresilienz bei Menschen voraussagen kann.

Ein Team um die UZH-Neuroökonomen Marcus Grüschow und Christian Ruff sowie UZH-Psychologin Birgit Kleim hat für die Studie eine Gruppe von MedizinstudentInnen untersucht, die alle kurz vor einer andauernden, realen Belastung standen - einem Praktikumshalbjahr in der Notaufnahme.

Kurz vor Praktikumsbeginn wurde den Probandlnnen eine Aufgabe gestellt, in der sie widersprüchliche Informationen verarbeiten mussten. Dabei wird der Bereich des Gehirns aktiviert, der die Reaktion in stressigen Situationen steuert: Das so genannte «Locus Coeruleus-Norepinephrine System» (LC-NE-Erregungs-System). Es hilft, den Konflikt zu überwinden. Die Intensität dieser Aktivierung unterscheidet sich jedoch von Mensch zu Mensch und wird «Feuerungsrate» genannt.

Probandlnnen, bei denen die Reaktion des LC-NE Systems stärker ausgeprägt war, zeigten nach dem Praktikum mehr Angst- und Depressionssymptome. Damit haben die Forschenden ein objektives, neurobiologisches Mass gefunden, das die Stressresilienz einer Person voraussagen kann. Als wohl erste humane Studie zeigt sie, wie Unterschiede in der Erregungsintensität des LC-NE-Systems als Indikator für Stressresilienz genutzt werden können.

Literatur: Marcus Grüschow et al. Predicting real-world stress resilience from the responsivity of the human locus coeruleus. Nature Communications. 15 April 2021. DOI: 10.1038/s41467-021-22509-1

Quelle: Universität Zürich (www.uzh.ch [15.04.2021]) 